\title{
Evaluation of spleen volume in thoracic computed tomography in patients with COVID-19 pneumonia
}

\author{
๑Cesur Samancı' ${ }^{1}$ ๑Bengü Saylan², ๑Gökçe Gülsen ${ }^{3}, \oplus$ Melike Yeşildal $^{4}$, ĐEyüp Çamurcuoğlu ${ }^{5}$, \\ (ㄷ)Fethi Emre Ustabaşığlu ${ }^{6}$ \\ ${ }^{1}$ Istanbul University Cerrahpaşa Medical Faculty, Radiology Department, Istanbul, Turkey \\ ${ }^{2}$ Haydarpaşa Sultan Abdülhamidhan Training and Research Hospital, Department of Pulmonary Medicine, Istanbul, Turkey \\ ${ }^{3}$ Haseki Training and Research Hospital, Department of Radiology, Ankara Turkey \\ ${ }^{4}$ Haydarpaşa Sultan Abdülhamidhan Training and Research Hospital, Department of Radiology, Istanbul, Turkey \\ ${ }^{5}$ University of Health Sciences, Sisli Hamidiye etfal Research Center, Department of Radiology, Istanbul, Turkey \\ ${ }^{6}$ Trakya University Medical Faculty, Radiology Department of Radiology, Edirne, Turkey
}

Cite this article as: Samancı C, Saylan B, Gülsen G, Gülsen G, Yeşildal M, Çamurcuoğlu E, Ustabaşığlu FE. Evaluation of spleen volume in thoracic computed tomography in patients with COVID-19 pneumonia. Anatolian Curr Med J 2021; 3(2); 93-97.

\begin{abstract}
Objectives: There is not enough data on the effect of COVID-19 on spleen volume in patients with COVID-19. Our aim was to compare the spleen volume of the COVID-19 patients with the control group.

Material and Method: 214 Patients (121 men, 93 women) who have a diagnosis of COVID-19 who have thorax CT were included in the study. In the patient group, there was evidence of viral pneumonia on thoracic CT or PCR was positive, all of them were patients who received treatment for Covid. The control group consisted of 185 patients (106 men, 79 women). Interobserver agreement was calculated. Analysis of the receiver operating characteristic (ROC) curve was used to identify a spleen volume cutoff value at which the accuracy for COVID-19 diagnosis was maximised.

Results: The mean spleen volume of the patient group was found to be 260 (range, 96.3-565, SD: 82.5) which was statistically significantly higher than the control group which was 220 (range, 125.9-331.9, SD: 34.5) (p<0.05). Excellent agreement was found between two blind observers and between intraobserver spleen volume measurements. In our study, the spleen volume for the diagnosis of COVID-19 pneumonia was 208.4 (AUC 0.639; 95\% CI 0.584-0.695), sensitivity and specificity were 69.6\% and $66.7 \%$, respectively.

Conclusions: Quantitative evaluation of spleen volume can give an idea about the Covid diagnosis. Spleen volume is higher in Covid group than control group.

Keywords: Coronavirus disease, COVID-19, computed tomography, splenomegaly
\end{abstract}

\section{INTRODUCTION}

Novel coronavirus disease 2019 (COVID-19) is an infectious disease that first appeared in Wuhan province, People's Republic of China (1). In the following process, this disease spread all over the world and was declared as a pandemic by the World Health Organization (WHO) (2). Although the gold standard for diagnosis is the real-time reverse transcription polymerase chain reaction (RT-PCR) test, thorax computed tomography (CT) examination stands out as a very important modality, especially in patients with clinical symptoms and negative results of the test (3). The spleen is the largest organ of the reticuloendothelial system. Increased spleen size (splenomegaly) is an important clinical finding especially for liver diseases and various diseases such as immune system, hematopoietic system, portal hypertension, splenic vein thrombosis and lymphoma. Therefore, it seems necessary to properly evaluate the size of the spleen, both in order to initiate a diagnostic process and to make appropriate treatment decisions and to follow the treatment response in certain cases $(4,5)$. Radiological imaging methods are widely used in the evaluation of spleen volume (6-9). In this study, it was aimed to measure the spleen size in thoracic $\mathrm{CT}$ of patients who were examined with non-contrast thorax CT with suspected COVID-19 and taking Covid treatment, to calculate the spleen volume and to evaluate the relationship between spleen volume and COVID-19. 


\section{MATERIAL AND METHOD}

\section{Patient Population}

Our study was approved by the Ümraniye Training and Research Hospital Ethics Committee (protocol number: B.10.1.TKH.4.34.H.GP.0.01). Patients who were examined with non-contrast thorax CT due to the suspicion of COVID-19 at the Haydarpaşa Sultan Abdulhamidhan Training and Research Hospital Radiology Department between April 4 and May 26 were retrospectively screened. Of the 360 patients found, 214 with radiological findings of infection or positive PCR were included in the study. 145 patients were excluded from the study. Of these 145 patients removed, 11 were not eligible for evaluation due to low image quality and 132 because the CT sections passing through the upper abdomen did not completely contain the spleen. Two patients were not included in the study due to their history of splenectomy. The control group consisted of 185 patients. All of them were patients who underwent thoracic CT for other reasons other than infection in 2019. In the patient group, there was evidence of viral pneumonia on thoracic CT or PCR was positive, all of them were patients who received treatment for Covid.

\section{Technical Data}

All patients were imaged in the supine position on 320 detector CT (Aquilion-ONE, Toshiba Medical Systems, Otawara, Japan). All images were obtained at $5 \mathrm{~mm}$ slice thickness in the lung window in the standard dose protocol. Standard protocol $(120 \mathrm{kVp}$, auto-mA - maximal $350 \mathrm{~mA}$ ) was used for thoracic CT scans. $\mathrm{CT}$ images were acquired at the end of the inspirium for a single breath-hold period. $1.25 \mathrm{~mm}$ collimation, 400 milliseconds $(\mathrm{msec})$ rotation, 1.35 pitch, 120 $\mathrm{kV}$ in X-ray tube and 400-480 milliamper/second (mAs), section thickness; $1.25 \mathrm{~mm}$ and reconstruction interval; Parameters of $2.5 \mathrm{~mm}$ were used. Field of view (FOV) was adjusted according to the size of the patient and a 512x512 matrix was used.

\section{Evaluation of Images}

Spleen index measurements in thoracic CT of the patients included in the study were made by two radiologists with 8 years and 9 years of professional experience. Both radiologists measured the spleen size unaware of each other's results. After both radiologists made the measurements separately, the 1st radiologist made all measurements again 15 days later. Measurements were made using picture archiving and communication system (PACS).

\section{Spleen Volume Calculation}

The methodology in the study by Kucybała et al. (10) was used to calculate the spleen volume. First of all in axial section (Figure 1a), maximal length (L) - the longest dimension between poles of the spleen, the thickness $(\mathrm{T})$ at the level of the hilum was obtained by measuring the distance between the inner and outer contours of the spleen. After that, the maximum height $(\mathrm{H})$ of the spleen was measured by measuring the distance between the upper and lower pole of the spleen in coronal reformatted sections (Figure 1b). Spleen volume was calculated using the formula of " $30+0.58$ (LxTxH)" and the range of 110-340 $\mathrm{mL}$ was accepted as the normal range, while the value above $340 \mathrm{ml}$ was evaluated as splenomegaly (10) (Figure 2).

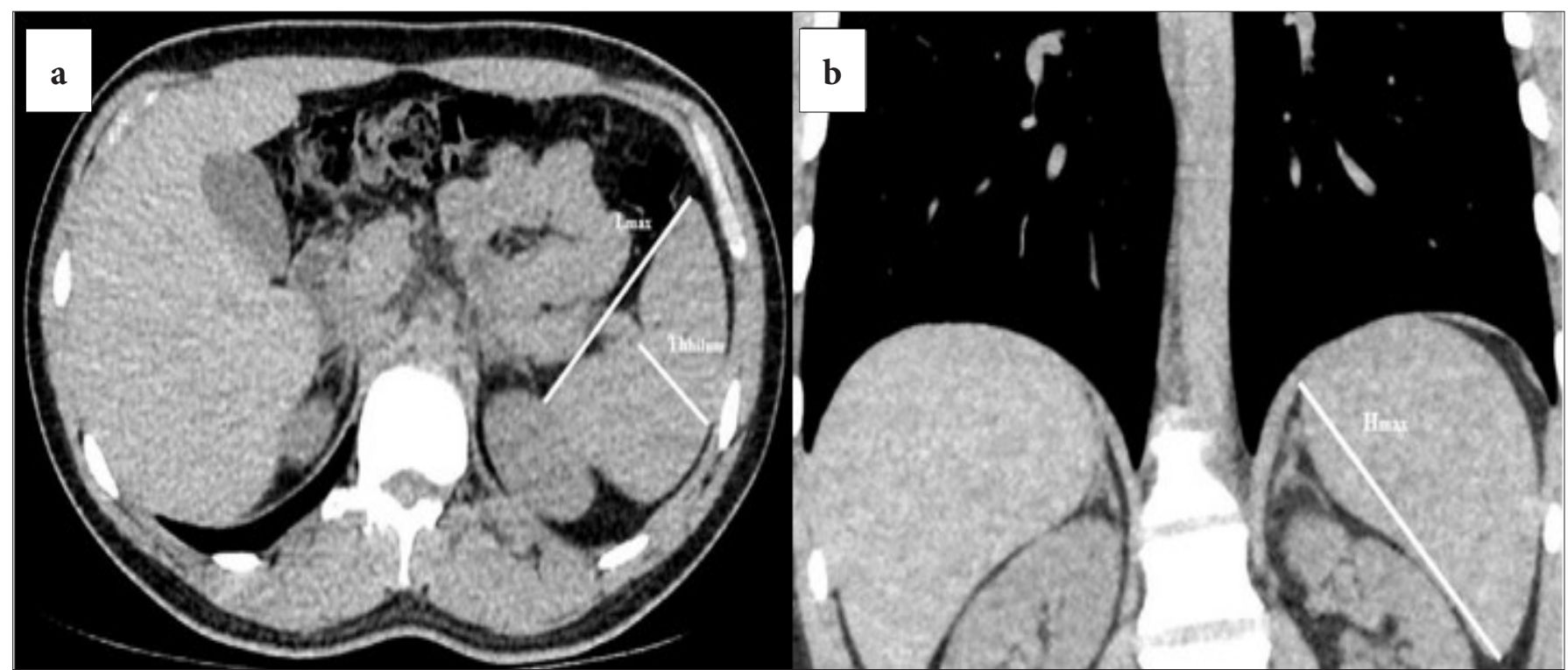

Figure 1. The method for calculating spleen volume on CT. First of all in axial section (a), maximal length (L)-the longest dimension between poles of the spleen, the thickness $(\mathrm{T})$ at the level of the hilum was obtained by measuring the distance between the inner and outer contours of the spleen. After that, the maximum height $(\mathrm{H})$ of the spleen was measured by measuring the distance between the upper and lower pole of the spleen in coronal reformatted sections (b). 

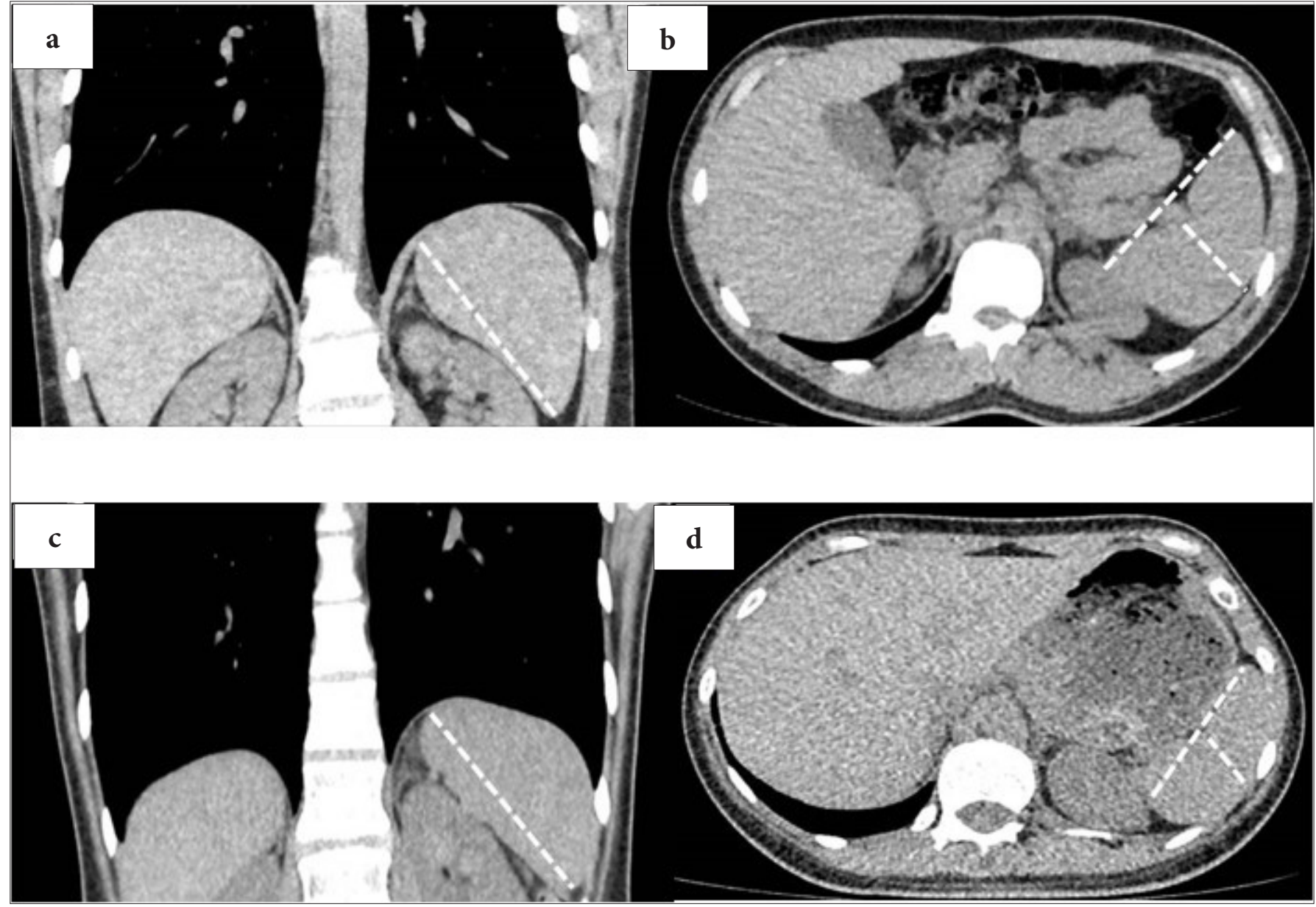

Figure 2. Spleen size in Covid-positive patient Hmax: 123mm Lmax: $98 \mathrm{~mm}$ Th: 50mm, Volum: 349mm3 (a-b), covid negative control group Hmax: $111 \mathrm{~mm}$ Lmax: $94 \mathrm{~mm}$ Th: $36 \mathrm{~mm}$ Volum $218 \mathrm{~mm} 3$ (c-d) in CT examination.

\section{Statistical Analysis}

Statistical Package for Social Science (SPSS, version 20.0) package program was used for statistical data analysis while evaluating the findings obtained in the study. Descriptive statistical methods were used while evaluating the study data. The results were evaluated at the $95 \%$ confidence interval and the significance level at $\mathrm{p}<0.05$. The data were evaluated using the KolmogorovSmirnov test, which showed a normal distribution. Comparison of the control group and the patient group by gender was calculated using the Chi-square test, and the comparison of BMI and spleen volume was calculated using the Mann Whitney U test. In the measurements, the intraclass correlation coefficient (ICC) was used to show inter-observer and intra-observer agreement. A ICC value greater than 0.80 indicates a perfect agreement. Correlations between variables were evaluated using the Pearson correlation coefficient.

\section{RESULTS}

Two hundred and fourteen Covid patients (121 men, 93 women) and 185 controls (106 men, 79 women) were included in this study. The mean age was 50.6 years (range, 18-86 years) for the patient group and 52.4 years (range, 21-79) for the control group. Body mass index was calculated as 26.8 (range, 20-30) in the patient group and 26.6 (range, 19.9-30) in the control group. In the Mann Whitney U test, no difference was found between age and BMI values, which are variables that can affect spleen size in the patient group and the control group. The mean spleen volume of the patient group was 260 (range, 96.3-565, SD: 82.5), and the mean spleen volume of the control group was 220 (range, 125.9-331.9, SD: 34.5). Comparing the two groups with the Mann Whitney U test, the mean of the patient group was found to be statistically significantly higher than the control group $(\mathrm{p}<0.05)$ (Table 1). Interobserver variability results are shown in (Table 2). Excellent agreement was found between two blind observers and between intraobserver spleen volume measurements. In our study, spleen volume for diagnosis of COVID-19 pneumonia was 208.4 (AUC 0.639 ; 95\% CI 0.584 0.695 ), sensitivity and specificity were $69.6 \%$ and $66.7 \%$, respectively shows the ROC curve plotted for splenic volume values (Figure 2). 


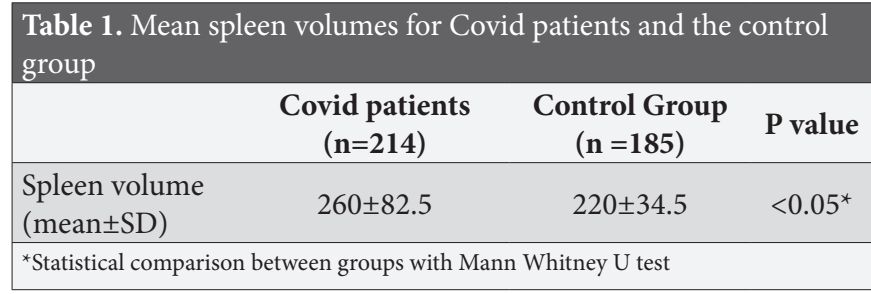

Table 2. Interobserver variability for spleen volume measurements

\begin{tabular}{|lccc|}
\hline & Observer 1 & Observer 2 & ICC \\
\hline & $($ mean \pm SD $)$ & $($ mean \pm SD $)$ & $\begin{array}{c}\text { intraclass correlation } \\
\text { coefficient }\end{array}$ \\
$\begin{array}{l}\text { Spleen volume } \\
(\mathrm{n}=399)\end{array}$ & $241.58 \pm 67.7$ & $241.76 \pm 67.1$ & $0.993(0.992-0.994)$ \\
\hline ICC: Intraclass Correlation Coefficients; SD: standard deviation & \\
\hline
\end{tabular}

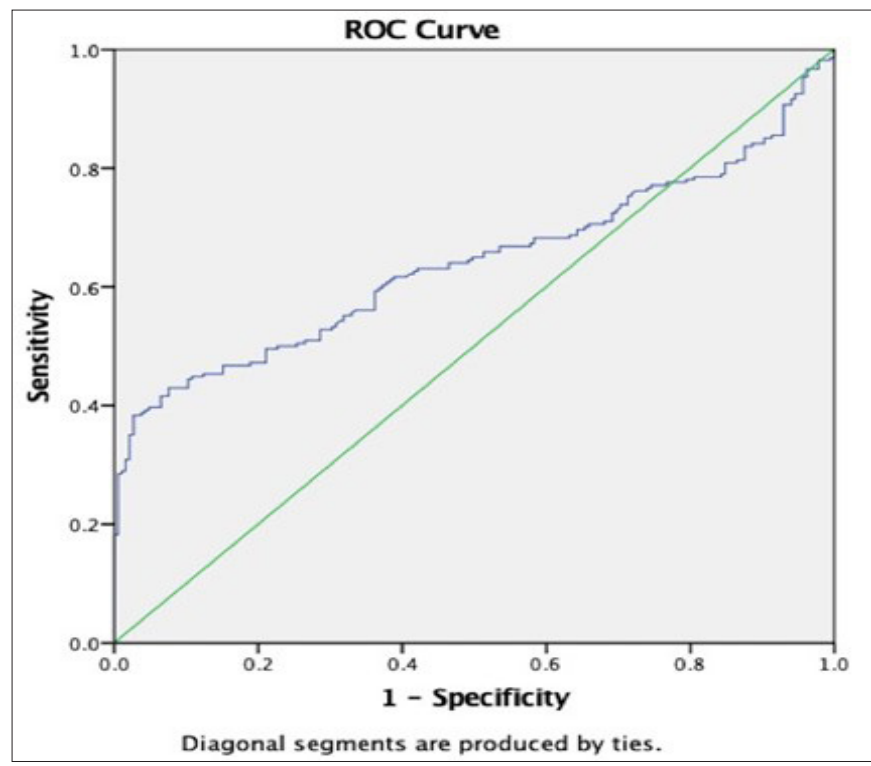

Figure 3. ROC curve for COVID-19 pneumonia diagnosis based on spleen volumes

\section{DISCUSSION}

The clinical features of COVID-19 range from asymptomatic to acute respiratory distress syndrome and multi-organ dysfunction. Common clinical features include fever (but not all), cough, sore throat, headache, fatigue, headache, myalgia, and shortness of breath. Conjunctivitis has also been described. Therefore, it cannot be distinguished from other respiratory infections. Sometimes at the end of the first week, the disease can progress to pneumonia, respiratory failure and death. The disease affects many systems $(11,12)$. When we look at the blood tests, the white cell count is usually normal or low. There may be lymphopenia; Lymphocyte count less than 1000 has been associated with severe disease. The platelet count is usually normal or slightly low (13). The spleen may be one of the organs directly attacked by the virus in some patients who die from COVID-19. T and $\mathrm{B}$ lymphocytes in the spleen are reduced to varying degrees, lymphoid follicles are reduced, and the number of NK cells does not change significantly. Pathological changes of the spleen may be related to the direct attack of the virus and the immune system attacking its own tissues. The literature shows that although other imaging techniques such as ultrasonography $(14,15)$ or magnetic resonance imaging (MRI) can be used, CT detects the varying splenic volume with the highest sensitivity and specificity (16). Additionally, a number of studies have demonstrated the significant accuracy of CT in the evaluation of the spleen in both pediatric and adult populations $(17,18)$. We also used CT in our study. Because of the suspicion of Covid pneumonia thoracic CT was already performed, an additional scan was not performed for spleen so no additional dose was given. In our study, we showed that the spleen volume increased statistically significantly in patients diagnosed with Covid, but the average did not exceed the limit of splenomegaly. Xu et al. (13) studied the pathological changes of the spleen in post-mortem COVID-19 patients and analyzed the relationship between the weakened immune system and splenic lesions. They showed that the cell composition of the spleen was decreased in histopathological examinations. Since the Covid pandemic is very new, there is not enough data in the literature on the relationship between Covid infection and spleen volume. While calculating spleen volume, agreement between observers was excellent which shows that the volume measurements based on CT is highly reproducible.

There were some limitations in our study. Although patients with known infections, hematological diseases, infiltrative diseases, and diseases of the spleen that may enlarge the spleen were excluded from the study, patients were not tested for these diseases. Therefore, other reasons that may cause splenomegaly may still be in the study. Another limitation was the small number of patients.

It would be useful to re-evaluate the spleen volumes after a certain period of time in order to determine the change in spleen volume over time. Randomized controlled studies with larger patient groups are needed to demonstrate the effect of infection on splenic volume in COVID-19 patients.

\section{ETHICAL DECLARATIONS}

Ethics Committee Approval: The research was approved by Ümraniye Training and Research Hospital Ethics Committee (protocol number: B.10.1.TKH.4.34.H.GP.0.01).

Informed Consent: Because the study was designed retrospectively, no written informed consent form was obtained from patients. 
Referee Evaluation Process: Externally peer-reviewed. Conflict of Interest Statement: The authors have no conflicts of interest to declare.

Financial Disclosure: The authors declared that this study has received no financial support.

Author Contributions: All of the authors declare that they have all participated in the design, execution, and analysis of the paper, and that they have approved the final version.

\section{REFERENCES}

1. Zhu N, Zhang D, Wang W, et al. A novel coronavirus from patients with pneumonia in China, 2019. N Engl J Med 2020; 382: 727-33.

2. Covid C, Team R. Severe outcomes among patients with coronavirus disease 2019 (COVID-19)_United States, February 12-March 16, 2020. MMWR Morb Mortal Wkly Rep 2020; 69: 343-6.

3. Xie X, Zhong Z, Zhao W, Zheng C, Wang F, Liu J. Chest CT for typical 2019-nCoV pneumonia: relationship to negative RT-PCR testing. Radiology 2020; 200343.

4. Wu WC, Chiou YY, Hung HH, et al. Prognostic significance of computed tomography scan-derived splenic volume in hepatocellular carcinoma treated with radiofrequency ablation. J Clin Gastroenterol 2012; 46: 789-95.

5. Strijk SP, Wagener DJ, Bogman MJ, de Pauw BE, Wobbes T. The spleen in Hodgkin disease: diagnostic value of CT. Radiology 1985; 154: 753-7.

6. Konus OL, Ozdemir A, Akkaya A, Erbas G, Celik H, Isik S. Normal liver, spleen, and kidney dimensions in neonates, infants, and children: evaluation with sonography. AJR Am J Roentgenology 1998; 171: 1693-8.

7. Loftus WK, Chow LTC, Metreweli C. Sonographic measurement of splenic length: correlation with measurement at autopsy. J Clin Ultrasound 1999; 27: 71-4.

8. Sandrasegaran K, Kwo PW, DiGirolamo D, Stockberger SM, Cum- mings OW, Kopecky KK. Measurement of liver volume using spiral CT and the curved line and cubic spline algorithms: reproducibility and interobserver variation. Abdominal Imaging 1999; 24: 61-5.

9. Schiano TD, Bodian C, Schwartz ME, Glajchen N, Min AD Accuracy and significance of computed tomographic scan assessment of hepatic volume in patients undergoing liver transplantation. Transplantation 2000; 69: 545-50.

10. Kucybała I, Ciuk S, Tęczar J. Spleen enlargement assessment using computed tomography: which coefficient correlates the strongest with the real volume of the spleen? Abdominal Radiology 2018; 43.9: 2455-61.

11. Chen N, Zhou M, Dong X, et al. Epidemiological and clinical characteristics of 99 cases of 2019 novel coronavirus pneumonia in Wuhan, China: a descriptive study. The Lancet 2020; 395: 50713.

12. Fan W, Su Z, Bin Y, et al. Holmes Edward C., Zhang Yong-Zhen. A new coronavirus associated with human respiratory disease in China. Nature 2020; 579: 265-9.

13. $\mathrm{Xu} \mathrm{X}$, Chang $\mathrm{XN}$, Pan $\mathrm{HX}$, et al. Pathological changes of the spleen in ten patients with coronavirus disease 2019 (COVID-19) by postmortem needle autopsy. Zhonghua bing li xue za $\mathrm{zhi}=$ Chinese journal of pathology 2020; 49: 576-82.

14. Lamb PM, Lund A, Kanagasabay RR, Martin A, Webb JAW, Reznek RH. Spleen size: how well do linear ultrasound measurements correlate with threedimensional CT volume assessments?. Br J Radiol 2002; 75: 573-7.
15. Elstein D, Hadas-Halpern I, Azuri Y, Abrahamov A, Bar-ziv Y, Zimran A. Accuracy of ultrasonography in assessing spleen and liver size in patients with Gaucher disease: comparison to computed tomographic measurements. J Ultrasound Med 1997; 16: 209-11.

16. Linguraru MG, Sandberg JK, Jones EC, Summers RM. Assessing splenomegaly: automated volumetric analysis of the spleen. Acad Radiol 2013; 20: 675-84.

17. Yetter EM, Acosta KB, Olson MC, Blundell K. Estimating splenic volume: sonographic measurements correlated with helical CT determination. AJR Am J Roentgenology 2003; 181: 1615-20.

18. Prassopoulos P, Cavouras D. CT assessment of normal splenic size in children. Acta Radiologica 1994; 35: 152-4. 